\title{
Evaluating Nursing Staff Mix in Long-Term Care: A Comprehensive Framework for Decision-Makers
}

Alice Kennedy

\begin{abstract}
The connection between care-provider type and patient outcomes is well established. Less well understood, however, is how to evaluate staff mix decision-making aimed at maximizing quality outcomes for care recipients, nursing staff and healthcare systems. From May through November 2008, Eastern Health, the largest regional integrated health authority in Newfoundland and Labrador, conducted a Staff Mix Intervention Project designed, in part, to gauge the effectiveness of the Evaluation Framework to Determine the Impact of Nursing Staff Mix Decisions in assessing the impact of nursing staff mix changes in long-term care. Results reveal that the Evaluation Framework is a useful tool for managers and other decision-makers to use when measuring the comprehensive effects of staff mix changes and planning further human resources modifications.
\end{abstract}

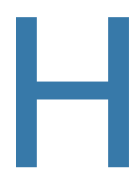

ealth human resources (HHR) is one of the principal concerns facing health system decisionmakers in Canada and throughout the developed world. As Nora Spinks and Celia Moore have recently

\section{“Effective HHR management is the cornerstone of a high-quality, efficient, publicly funded healthcare system."}

argued, "Effective HHR management is the cornerstone of a high-quality, efficient, publicly funded healthcare system" (2007: 38). Ensuring this solid foundation can be hugely challenging, however, in the context of an aging and shrinking labour force, a greying population and budgetary constraints.

In the nursing sector, a great deal of professional and scholarly attention has been paid to the HHR issue in the form of nursing staff mix: the combination and number of regulated and unregulated persons providing direct and indirect nursing care where regulated nursing groups practise (Canadian Nurses Association 2003). This subject has received such a high degree of scrutiny largely because of the well-documented connections between patient outcomes and safety and the numbers, proportions and types of nurses delivering care. Researchers have found, for example, several significant benefits in hospitals that have more nurses per patient or a higher proportion of registered nurses (RNs). Benefits include decreased mortality rates; lower rates of hospital readmission in the 30 days after discharge; shorter lengths of stay; fewer incidents of pressure ulcers, urinary tract infections and postoperative infections; and greater patient satisfaction with care (Aiken et al. 1999, 2002; 
American Nurses Association 1997, 2000; Hunt and Hagen 1998; McGillis Hall et al. 2003, 2004; Needleman et al. 2002; O'Brien-Pallas et al. 2001; Person et al. 2004; Sovie and Jawad 2001; Tourangeau et al. 2002).

This wealth of evidence has contributed to calls for employers to optimize their nursing staff mixes (e.g., Baumann et al. 2001). While it is understood that staffing decisions must take into account nurses' experience, an organization's unique characteristics and the quality of collaboration among all levels of employees within a given facility (Curtin 2003), managers and other decision-makers in individual facilities, regional health authorities and government ministries struggle to comprehend concretely the impact of various configurations of regulated nursing personnel and unregulated care providers. This knowledge shortfall is largely the result of a lack of literature on the evaluation of nursing staff mix decision-making (Cho et al. 2003; Jawad et al. 2003; Needleman et al. 2002; Yang 2003).

At Eastern Regional Integrated Health Authority (www. easternhealth.ca), the largest regional integrated health authority in Newfoundland and Labrador (NL) and Atlantic Canada, we recently undertook the Staff Mix Intervention Project. This six-month study investigated the impact and evaluation of nursing staff mix changes implemented in three of our longterm care (LTC) facilities. I served as the principal investigator on this project as part of my fellowship in the Canadian Health Services Research Foundation's Executive Training for Research Application program (www.chsrf.ca/extra/).

From a management perspective, one of our major objectives was to gauge the utility of the Evaluation Framework to Determine the Impact of Nursing Staff Mix Decisions for assessing the impact of staff mix changes on LTC residents, staff and the system (Figure 1). The Canadian Nurses Association (CNA) published the framework in 2005. It had been developed by CNA in collaboration with the Canadian Practical Nurses Association, the Canadian Council for Practical Nurse Regulators and Registered Psychiatric Nurses of Canada. Eastern Health's Staff Mix Intervention Project involved the first test of this instrument in an LTC context.

In this article, I outline the provincial and regional budgetary and HHR contexts in which the Staff Mix Intervention Project took place, and follow with an explanation of the personnel changes we made and the methods we used to collect outcomes data. I then address the principal results as they pertain to the evaluation of staff mix decision-making, and conclude with thoughts on the implications of our study for managerial decision-making.

\section{Contexts}

\section{Provincial Context}

The government of NL reduced funding to the province's LTC facilities in its 2004 budget. At the same time, the government challenged the LTC system to become more efficient with respect to nursing staff mix by decreasing the number of RNs and licensed practical nurses (LPNs), as well as by enabling these professionals to work to their full scopes of practice (i.e., the range of services a professional group is authorized to provide [College of Registered Nurses of Manitoba 2002]). While NL traditionally has had relatively few personal care attendants (PCAs) working in its healthcare system, the government urged the LTC system to increase significantly the number of these unregulated health workers.

In February 2005, a review of peer sites from other provinces showed that nursing homes in NL exceeded critical measures seen elsewhere in the country:

- The highest nursing staff mix in Canada (RNs: $22-30 \%$; LPNs: 70-78\%)

- Some of the highest hours of care per resident in the country (from 2.5 to 4.7 hours)

- One of the highest cost-per-resident levels (from $\$ 6,000$ to $\$ 7,000$ per resident per month)

Likewise, when the roles and responsibilities of RNs and LPNs in NL were compared with their peers in other parts of Canada, it was found that RNs in NL were focusing on tasks that LPNs elsewhere were handling (e.g., medication administration). We also learned that LPNs in NL were performing tasks most often undertaken by PCAs in other provinces. Scopeof-practice shortcomings were felt particularly acutely by recent LPN graduates who had been trained to administer medication and by those who had also received training in health assessment. The normative staff mix in NL's LTC facilities, however, generally did not allow medication- and assessmentproficient LPNs to work to their full scope of practice. In cases in which employers made such work possible, many RNs reacted negatively, regarding these new LPN functions as encroachments on the RNs' role. Given that NL's College of Licensed Practical Nurses has mandated that by April 2012 all LPNs will be required to have completed the medication administration and health assessment courses, coupled with the difficulty of recruiting RNs and LPNs to work in the LTC sector, the need to address nursing staff mix has never been more urgent.

In November 2005, the NL Department of Health and Community Services formed a Staff Mix Committee to move forward the implementation of a new skill mix and staffing ratio in the province's LTC sector and to introduce PCAs into nursing homes. The committee released its report a year later; among the service-delivery changes it recommended were the following interdisciplinary team ratios:

- Lower the RN staff mix ratio to between 14 and $20 \%$

- Lower the LPN staff mix ratio to between 40 and 50\% 


\section{Figure 1. Evaluation Framework to Determine the Impact of Nursing Staff Mix Decisions}

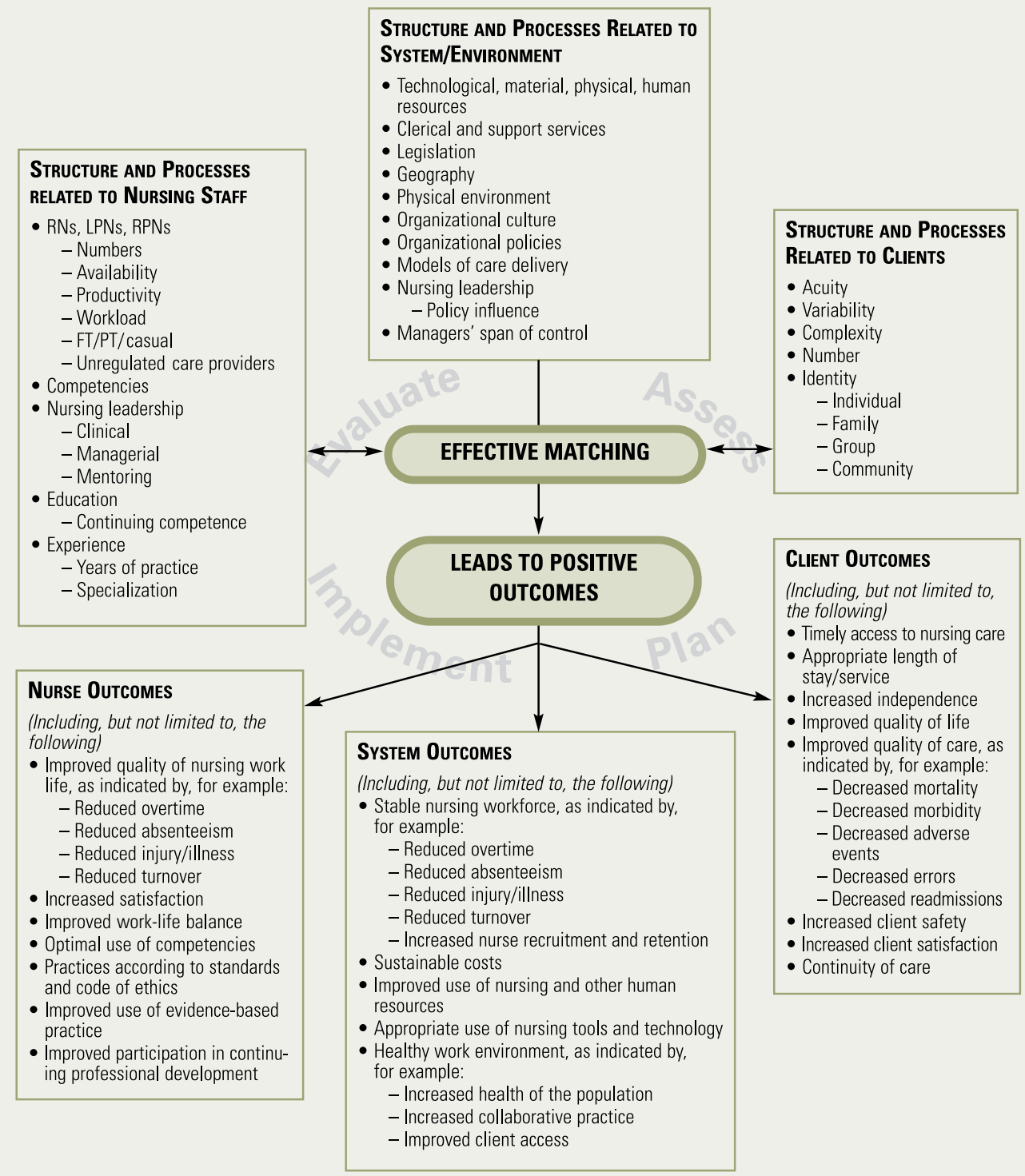

$\mathrm{FT}$ = full time; $\mathrm{PT}=$ part time; $\mathrm{LPN}=$ licensed practical nurse; $\mathrm{RN}=$ registered nurse; RPN = registered practical nurse

Source: Canadian Nurses Association (2005).

- LPNs: comprehensive health assessor and medication administrator

- PCAs: provider of direct care, support and companionship under the direction of an RN or LPN

Finally, the Staff Mix Committee observed the importance of ongoing evaluation of personnel changes in order "to ensure the new staff mix is meeting the care needs of the residents and to ensure successful transition to the new roles for care providers" (Provincial Committee to Review Staff Mix in Long Term Care Settings 2006: 14). To that end, the committee endorsed use of the Evaluation Framework.

\section{Eastern Health Context}

Eastern Health was formed in April 2005 from the merger of seven healthcare boards. This regional integrated health authority employs more than 12,000 people, has a budget of over $\$ 1$ billion and provides a full range of

- Increase the PCA staff mix ratio to between 33 and $40 \%$

The Staff Mix Committee also provided descriptions for each of the three care providers' new scopes of practice:

- RNs: practitioner, leader, patient care coordinator, program planner, educator healthcare services to a population of over 290,000. Altogether, our 13 LTC facilities have 1,450 beds, approximately $65 \%$ of NL's total.

Faced with a growing need for high-level LTC beds and a diminishing and aging nursing workforce, Eastern Health opted to follow the Staff Mix Committee's recommendations regarding changing the numbers and ratios of the regulated 
nursing personnel and unregulated PCAs employed in its LTC facilities. Early in our planning, we recognized that we needed a new model of care delivery and that the interventions we envisioned would entail a major change in how LTC care is provided and would have significant implications for HHR, labour relations and education and training. We also knew that providing evidence to support the changes would be critical to obtaining the support of all our internal and external stakeholders - among the most important, residents and their families, RNs, LPNs, PCAs and staff unions. Just as important, we wanted to be able to assess unambiguously the impact of the changes we made so as to support further evidence-based staff mix decision-making both at Eastern Health and across NL's other three regional authorities. These considerations all contributed to our adoption of the Evaluation Framework as a suitable tool for measuring our personnel changes and supporting future planning.

\section{Methods}

From May through November, we implemented the Staff Mix Intervention Project at three Eastern Health LTC sites - two urban (St. Patrick's Mercy Home and Hoyles-Escasoni Complex, both in St. John's) and one rural (Golden Heights Manor, in Bonavista). At each site, we designated one unit a pilot unit and one unit a non-intervention control unit. In making our selections, we ensured that residents living in the pilot units had levels of acuity similar to those in the control units. Table 1 gives the number of residents in each unit during the study.

In the pilot units, we changed the care delivery model to take advantage of the potential full scopes of practice of RNs and LPNs. PCAs were also introduced into the personnel mix or, where they already existed, their numbers were significantly increased (Table 2). Staff mixes on the control units were unaltered.

We developed and implemented a slate of data-gathering measures that coincided with the three outcomes categories used in the Evaluation Framework: client outcomes (the Evaluation
Framework uses the term client to refer to any recipient of nursing care - in the Staff Mix Intervention Project, our clients were residents of the three LTC facilities); nurse outcomes (while we adhered to the Evaluation Framework's "nurse outcomes" rubric to organize our data collection and analysis, we included PCAs in this category); and system outcomes (Table 3). The Evaluation Framework provided a comprehensive list of factors to consider when identifying indicators that impact staff, client and system outcomes. These measures and the study itself received ethics approval from the Human Investigation Committee of the Faculty of Medicine at Memorial University of Newfoundland and Labrador.

Table 1. Number of residents living in the pilot and control units at the three LTC facilities

\begin{tabular}{|l|l|}
\hline LTC Facility & Number of Residents \\
\hline St. Patrick's Mercy Home & $\begin{array}{l}\text { Pilot unit: 44 } \\
\text { Control unit: } 44\end{array}$ \\
\hline Hoyles-Escasoni Complex & $\begin{array}{l}\text { Pilot unit: } 25 \\
\text { Control unit: } 21\end{array}$ \\
\hline Golden Heights Manor & $\begin{array}{l}\text { Pilot unit: } 40 \\
\text { Control unit: } 30\end{array}$ \\
\hline
\end{tabular}

LTC = long-term care

\section{Data Gathering for Client Outcomes}

Residents who were considered cognitively well were identified and approached by the resident care managers or social workers at each facility to participate in the resident satisfaction survey, which was divided into nine sections: living environment, activities, choice, communication, care and services, assistance with eating, overall environment, recommending the facility and

Table 2. Average nurse mix staffing ranges and hours of care per day in the pilot units

\begin{tabular}{|l|c|c|c|}
\hline & St. Patrick's Mercy Home & Hoyles-Escasoni Complex & Golden Heights Manor \\
\hline RN range average (\%) & 16 & 17.4 & 14 \\
\hline LPN range average (\%) & 32 & 34.8 & 48 \\
\hline PCA range average (\%) & 52 & 47.8 & 38 \\
\hline Average hours of care per day & 3.15 & 3.5 & 3.1 \\
\hline
\end{tabular}

LPN = licensed practical nurse; $\mathrm{PCA}=$ personal care attendant; $\mathrm{RN}=$ registered nurse 
Table 3. Methods and resources used to gather data for the Evaluation Framework's three outcomes
categories

\begin{tabular}{|c|c|c|c|}
\hline Data-Gathering Methods and Resources & Client Outcomes & Nurse Outcomes & System Outcomes \\
\hline $\begin{array}{l}\text { Resident/family and nursing staff satisfaction surveys (pre-implementation } \\
\text { and during project) }\end{array}$ & $x$ & $X$ & \\
\hline Focus groups & & $x$ & \\
\hline Systematic tracking of compliments, complaints and care-quality indicators & $x$ & & \\
\hline Human resources data & & $x$ & $x$ \\
\hline Informal staff feedback & & $x$ & $x$ \\
\hline Technology-enabled audits using minimal data set tool & & & $x$ \\
\hline Informal resident, family and staff feedback & $X$ & & $x$ \\
\hline
\end{tabular}

location at the facility. Fifty-two residents in total were identified to participate, and the interviews, most of which lasted approximately 30 minutes, were administered by a research assistant in a quiet room. We also conducted family satisfaction surveys via telephone interviews. In total, 189 family members were contacted. Finally, we tracked compliments, complaints and quality-of-care indicators (e.g., medication errors, adverse events, falls, restraints, infections and pressure ulcers). The latter data are regularly monitored in each of Eastern Health's LTC facilities, thereby giving us valuable baseline data for our analysis.

\section{Data Gathering for Nurse Outcomes}

Our first step was to hold focus group sessions with nursing staff members (primarily LPNs but also including RNs and PCAs) at the participating facilities. We used the information from these meetings to help develop the staff satisfaction survey and to aid our interpretation of the study's findings.

The staff satisfaction survey contained 86 items. The first section (29 items) was adapted from Karasek's Job Content Questionnaire, which is used extensively to assess stress and strain in work environments (Bourbonnais et al. 1999; Hellerstedt and Jeffery 1997; Karasek 1985, 1998). Much of the second section (47 items) was adapted from the 2005 National Survey of the Work and Health of Nurses (NSWHN) (Shields and Wilkins 2006); this part assessed various job characteristics specific to the nursing profession. In addition to items drawn from the NSWHN, we included four items that elicited participants' opinions regarding the current staffing mix. The third and final section included 10 demographic items (e.g., profession, age, gender, length of time employed at any Eastern Health sites). In total, 155 surveys were distributed to staff at the three facilities taking part in our study.
Finally, we also collected human resources data, including rates on absenteeism, injury/illness and turnover. Because each of the sites uses a different payroll system, we tracked the units' costs via the individual full-time equivalents using the scheduling packages at each site.

\section{Data Gathering for System Outcomes}

Using Eastern Health's payroll data, we collected and monitored system outcomes, such as cost per resident per day per unit, as well as human resources indicators, such as overtime, absenteeism, injury/illness, turnover and nurse recruitment and retention. These data enabled us to detect whether the altered staff mixes affected labour costs and adverse events. We also audited the use of technology (e.g., the minimal data set [MDS] tool), and, in order to assess the health of the units' work environments and the stability of the nursing workforce, we reviewed staff feedback on collaborative practice as well as feedback from residents on access to services.

\section{Outcomes}

In this section, I describe selected results of our Staff Mix Intervention Project in terms of the light they shed on the utility of the Evaluation Framework. At a later date, I will prepare a full technical report addressing all the project's outcomes.

\section{Client Outcomes}

The Evaluation Framework allowed us to organize and analyze evidence that revealed there were no significant differences attributable to staff mix changes in the health outcomes among residents on the pilot and control units. Likewise, residents and their family members on the two sets of units expressed similarly positive views of the care delivered. 
In the initial phase of the project, however, pilot-unit residents informed us that they had to wait longer to access nursing care (e.g., to be fed and bathed). We quickly addressed this shortcoming by adding extra PCA hours at peak care times. Staff also reported that residents' care needs could be met more swiftly on the pilot units if their medications arrived in a more timely fashion.

Toward the last month of the intervention, pilot-unit residents had more favourable perceptions of care, service and overall environment. The quality-of-care outcomes we examined revealed inconsistent patterns and illustrated a need for changes in reporting procedures. There was no evidence that the new staff mix negatively impacted residents' care.

\section{While RNs on the control units} continued to feel overwhelmed by the level of their responsibilities, pilot-unit RNs took on leadership roles and embraced the opportunity to engage in higher-level nursing functions.

\section{Nurse Outcomes}

On one of the pilot units, RNs initially expressed concern that they were no longer participating to their full scope of practice. As a result, they felt out of touch with the residents. By the end of the project, however, RNs, LPNs and PCAs on the pilot units were claiming greater job satisfaction than were their colleagues on the control units. While RNs on the control units continued to feel overwhelmed by the level of their responsibilities, pilotunit RNs took on leadership roles and embraced the opportunity to engage in higher-level nursing functions (e.g., resident assessment, critical thinking, prioritizing and directing care).

There were no overall differences found among responses regarding staff perception of work autonomy, control over practice, roles, respect, support and communications between the beginning and the end of the intervention project. It is interesting, however, that staff on the control units were more likely to report that the staff mix on their units needed to be changed and perceived the pilot mix as better. A notable change in the number of staff reporting an overall improvement in the perceived quality of resident care increased from $9 \%$ in phase one to $21 \%$ in phase two. Meanwhile, pilot-unit LPNs and PCAs noted feeling "empowered" by being more involved in residents' care: LPNs worked to their full scope and administered medication, and they were paired with PCAs, who ensured care was provided to assigned residents.

\section{System Outcomes}

Staff mix changes led to perceptions of an improved work environment on the pilot units, which arose primarily as a result of dismantling traditional workplace hierarchies and by allowing staff to work to their full scopes of practice. We predict that this systemic change will improve the HHR situation by enhancing the attractiveness of the LTC sector for both RNs and LPNs, most of whom tend to gravitate to careers in acute care.

The control units, although appropriately staffed, struggled during times when new staff members were required to provide relief (e.g., when staff were on vacation or long-term leave). This problem was compounded by the systemic HHR difficulty of recruiting staff to the LTC sector; at times, in fact, RN and LPN staffing levels on the control units were lower than targeted and, indeed, almost at pilot-unit staffing levels. Meanwhile, the pilot units' mixes enabled greater flexibility in dealing with staff shortages.

Analysis of variations in overtime, absenteeism and sick leave between the control and intervention units within sites pre- and post-implementation showed no consistent patterns. This may be attributed to the small sample sizes used in this pilot study. However, the findings are positive in that the new staff mix did not appear to influence rates of overtime, absenteeism or sick leave - all indicators of job satisfaction.

In the early stages of the project, pilot units struggled with a lack of equipment - in particular, a shortage of medication carts and computer access. This problem was quickly addressed by obtaining extra carts and computers. Again, the ongoing feedback from residents, staff and families was considered and responded to as part of our use of the Evaluation Framework. These steps are inherent in the framework's matching of structures and processes with outcomes and ongoing evaluation.

\section{Discussion}

The responsibility for ensuring appropriate nursing staff mix rests with nurse administrators and managers, including supervisors as well as middle and senior managers (CNA 2003). Integral to fulfilling that responsibility is the ability to assess the impacts of changes made to personnel deployment configurations, and this relies on the collection and organization of high-quality, relevant, evidence-based outcomes data.

In the case of Eastern Health's Staff Mix Intervention Project, the Evaluation Framework enabled us to accomplish these goals in ways that contributed to our overall goal of shaping an optimal future for our LTC residents, nurses and the system itself. It did so largely by focusing attention on the fundamental elements affecting resident, staff and system outcomes. In the words of Rod Hayward, an LPN and unit manager at the HoylesEscasoni Complex, "Using the Evaluation Framework made me cognizant that RNs were not optimally utilized outside the pilot 
site, and that having LPNs and PCAs working in tandem generates greater job satisfaction and workplace efficiency."

As we reflect on the project's outcomes in relation to our use of the Evaluation Framework, it is clear that this measurement and decision-making tool is best deployed as part of a transparent, workforce-wide embrace of evidence-informed practice. To that end, healthcare administrators engaged in nursing staff mix change would be well advised to invest resources - personnel and financial - in knowledge transfer and infrastructure support. Moving through the change process requires unit-level change agents or team leaders, as well as strategies that empower staff to increase their morale and job satisfaction. In this regard, the Evaluation Framework can be a valuable component of managing resistance to change, in particular by channelling and validating input from front-line staff.

On a more tactical level, when developing target staff mixes for units and facilities, the Evaluation Framework offers a pragmatic resource for identifying key areas related to structure and processes that require planning consideration. In turn, those issues help to guide the implementation of new staff mixes. The Evaluation Framework also supports the ongoing evaluation and corollary adaptation of staff mix plans, all aimed at achieving positive client, staff and system outcomes. By using the Evaluation Framework in our project, we were able to monitor and adjust our plans according to emergent staff mix problems; this tool also gave us concrete evidence on which we drew to swiftly correct these problems. In this respect, as I mentioned earlier, combined resident and staff feedback regarding care-delivery delays prompted us in the first month of our project to add PCA resources during a peak-demand period.

There is no single staff mix number or ratio applicable across the board. Configurations must be correlated to variables such as residents' acuity levels, staff competency and the physical layout of the unit or facility. Because of its inherent flexibility, the Evaluation Framework supports making, assessing and revising decisions that are in the best interests of local stakeholders and the facility. As Eastern Health rolls out revised nursing staff mixes across its entire LTC system, managers and decisionmakers will, we are confident, rely on the Evaluation Framework as a comprehensive HHR planning and assessment tool.

\section{Lessons Learned}

Using the Evaluation Framework taught us a number of lessons that will inform staff mix changes in other Eastern Health LTC units and facilities. Foremost among those lessons is the critical importance of ensuring that resources are provided to plan and implement staff mix changes and related data collecting to evaluate clearly and systematically impacts at the client, staff and system levels. Providing information in multiple forms on a continuous basis is also necessary to ensure that the people affected fully understand the rationale for the staff mix changes. In addition, implementing a change where there is no body of evidence to support the initiative has implications for the health system as a whole and requires broad participation of internal and external stakeholders at the regional, provincial and national levels. In this regard, having a framework that generates supporting evidence is essential for the success of such initiatives in every aspect.

\section{Limitations}

The results of the Staff Mix Intervention Project are robust and will contribute strongly to future staff mix decision-making and evaluation activities in Eastern Health and NL's other three regional integrated health organizations. We acknowledge, however, that certain structural difficulties presented challenges to our work.

Making optimal use of the Evaluation Framework relies on consistently rendered resident, staff and system data across all sites. At the data-collection stage, however, we had some difficulty retrieving baseline statistics because the seven previously independent boards comprising Eastern Health have not yet fully amalgamated their information recording and reporting methods. Also, limitations existed regarding the data generated, particularly concerning quality-of-care and human resource indicators. Inconsistencies found in the quality-of-care indicators were defined and reported between sites.

Given the fluctuating nature of human health, it was also impossible to control absolutely for levels of acuity among residents. Similarly, staffing ratios changed on a daily basis on both the pilot and control units. For instance, RNs, LPNs and PCAs took their scheduled vacations and, on occasion, were absent due to illness. However, each unit strove to find temporary staff to mitigate the effects of staff shortages on residents. Nevertheless, due to staffing shortages, some staff worked on both pilot and control units. As well, the fact that in Eastern Health some staff work 12-hour shifts while others work eighthour shifts complicated our assessment activities. In all cases, however, we believe the Evaluation Framework proved sufficiently sensitive to enable us to factor in these variables when we were analyzing the outcomes data.

\section{Acknowledgement}

I gratefully acknowledge the support provided by the Executive Training for Research Application Program administered by the Canadian Health Services Research Foundation in collaboration with a group of partnering organizations: the Canadian College of Health Service Executives, CNA, the Canadian Medical Association and a consortium of 12 Quebec partners, represented by the Agence des technologies et des modes d'intervention en santé. I also acknowledge the support 
of Eastern Health's Department of Corporate Strategy and Research, the Department of Psychology at Memorial University of Newfoundland and Labrador and CNA. HQ

\section{References}

Aiken, L.H., D.M. Sloane, E.T. Lake, J. Sochalski and A.L. Weber. 1999. "Organization and Outcomes of In-Patient AIDS Care." Medical Care 37(8): 760-72.

Aiken, L.H., S.P. Clarke and D.M. Sloane. 2002. "Hospital Staffing, Organization, and Quality of Care: Cross-National Findings." International Journal for Quality in Health Care 14(1): 5-13.

American Nurses Association. 1997. Implementing Nursing's Report Card: A Study of RN Staffing, Length of Stay and Patient Outcomes. Washington, DC: Author.

American Nurses Association. 2000. Nurse Staffing and Patient Outcomes in the Inpatient Hospital Setting. Washington, DC: Author.

Baumann, A.L., L. O’Brien-Pallas, M. Armstrong-Stassen, J. Blythe, R. Bourbonnais, S. Cameron, D. Irvine-Doran, M. Kerr, L. McGillisHall, M. Vézina, M. Butt and L. Ryan. 2001. Commitment and Care: The Benefits of a Healthy Workplace for Nurses, Their Patients and the System. Ottawa, ON: Canadian Health Services Research Foundation and the Change Foundation. Retrieved December 10, 2008. <http:// www.chsrf.ca/funding_opportunities/commissioned_research/polisyn/ pdf/pscomcare_e.pdf $>$.

Bourbonnais, R., M. Comeau and M. Vézina. 1999. "Job Strain and Evolution of Mental Health among Nurses." Journal of Occupational Health Psychology 4: 95-107.

Canadian Nurses Association. 2003. Position Statement: Staffing Decisions for the Delivery of Safe Nursing Care. Ottawa, ON: Author. Retrieved December 10, 2008. <http://www.cna-aiic.ca/CNA/ documents/pdf/publications/PS67_Staffing_Decisions_Delivery_ Safe_Nursing_Care_June_2003_e.pdf $>$.

Canadian Nurses Association, Canadian Practical Nurses Association, Canadian Council for Practical Nurse Regulators and Registered Psychiatric Nurses of Canada. 2005. Evaluation Framework to Determine the Impact of Nursing Staff Mix Decisions. Ottawa, ON: Canadian Nurses Association. Retrieved December 10, 2008. <http:// www.cna-aiic.ca/CNA/documents/pdf/publications/Evaluation Framework_2005_e.pdf>.

Cho, S.-H., K. Ketefian, V.H. Barkauskas and D.G. Smith. 2003. "The Effects of Nurse Staffing on Adverse Events, Morbidity, Mortality, and Medical Costs." Nursing Research 52(2): 71-79.

College of Registered Nurses of Manitoba. 2002. Guidelines for Decision-Making Regarding the Appropriate Nursing Care Provider. Winnipeg, MN: Author.

Curtin, L.L. 2003. "An Integrated Analysis of Nurse Staffing and Related Variables: Effects on Patient Outcomes." Online Journal of Issues in Nursing 8(3). Retrieved December 10, 2008. <http://www.nursingworld.org/MainMenuCategories/ANAMarketplace/ANAPeriodicals/ OJIN/KeynotesofNote/StaffingandVariablesAnalysis.aspx>.

Hellerstedt, W.L. and R.W. Jeffery. 1997. "The Association of Job Strain and Health Behaviours in Men and Women." International Journal of Epidemiology 26: 575-83.

Hunt, J. and S. Hagen. 1998. "Nurse to Patient Ratios and Patient Outcomes.” Nursing Times 11(94): 63-66.
Jawad, A.F., C.C. Scalzi and T. Sasicha-Akkadechanunt. 2003. "The Relationship between Nurse Staffing and Patient Outcomes." Journal of Nursing Administration 33(9): 478-85.

Karasek, R.A. 1985. Job Content Questionnaire and User's Guide. Lowell, MA: University of Massachusetts Lowell, Department of Work Environment.

Karasek, R.A., C. Brisson, N. Kawakami, I. Houtman, P. Bongers and B. Amick. 1998. "The Job Content Questionnaire (JCQ): An Instrument for Internationally Comparative Assessments of Psychosocial Job Characteristics." Journal of Occupational Health Psychology 3: 322-55.

McGillis Hall, L., D. Doran and G.H. Pink. 2004. "Nursing Staff Mix Models, Nursing Hours and Patient Safety Outcomes." Journal of Nursing Administration 34(1): 41-45.

McGillis Hall, L., D. Doran, G.R. Baker, G.H. Pink, S. Sidani, L. O'Brien-Pallas and G.J. Donner. 2003. "Nurse Staffing Models as Predictors of Patient Outcomes." Medical Care 41(9): 1096-109.

Needleman, J., P. Buerhaus, S. Mattke, M. Stewart and K. Zelevinsky. 2002. "Nurse-Staffing Levels and the Quality of Care in Hospitals." New England Journal of Medicine 346(22): 1715-22.

O’Brien-Pallas, L., D. Thomson, C. Alksnis and S. Bruce. 2001. “The Economic Impact of Nurse Staffing Decisions: Time to Turn Down Another Road?" Hospital Quarterly 4(3): 42-50.

Person, S.D., J.J. Allison, C.I. Kiefe, M.T. Weaver, O.D. Williams, R.M. Centor and N.W. Weissman. 2004. "Nurse Staffing and Mortality for Medicare Patients with Acute Myocardial Infarction." Medical Care 42(1): 4-12.

Provincial Committee to Review Staff Mix in Long Term Care Settings. 2006. Final Report of the Provincial Committee to Review Staff Mix in Long Term Care Settings. St. John's, NL: Newfoundland and Labrador Department of Health and Community Services.

Shields, M. and K. Wilkins. 2006. Findings from the 2005 National Survey of the Work and Health of Nurses. Ottawa, ON: Health Canada and Canadian Institute for Health Information. Retrieved December 11, 2008. <http://secure.cihi.ca/cihiweb/products/NHSRep06_ENG.pdf>.

Sovie, M.D. and A.F. Jawad. 2001. "Hospital Restructuring and Its Impact on Outcomes: Nursing Staff Regulations are Premature." Journal of Nursing Administration 31(12): 588-600.

Spinks, N. and C. Moore. 2007. "The Changing Workforce, Workplace and Nature of Work: Implications for Health Human Resource Management.” Nursing Leadership 20(3): 26-41.

Tourangeau, A.E., P. Giovannetti, J.V. Tu and M. Wood. 2002. "Nursing-Related Determinants of 30-Day Mortality for Hospitalized Patients." Canadian Journal of Nursing Research 33(4): 71-88.

Yang, K.-P. 2003. "Relationships between Nurse Staffing and Patient Outcomes." Journal of Nursing Research 11(3): 149-57.

\section{About the Author}

Alice Kennedy, BN, RN, MBA, CHE, FCCHSE, is the Chief Operating Officer of long-term care, community living and supportive services, palliative care, continuing care and rehabilitation with the Eastern Regional Integrated Health Authority in Newfoundland. 